Moroccan J. of Pure and Appl. Anal. (MJPAA)

Volume 7(3), 2021, Pages 364-374

ISSN: Online 2351-8227 - Print 2605-6364

DOI: $10.2478 / \mathrm{mjpaa}-2021-0024$

\title{
Integral geometry on discrete matrices
}

\author{
Abdelbaki Attioui ${ }^{1}$
}

Aвstract. In this note, we study the Radon transform and its dual on the discrete matrices by defining hyperplanes as being infinite sets of solutions of linear Diophantine equations. We then give an inversion formula and a support theorem.

Mathematics Subject Classification (2020). Primary: 44A12, 44A53; Secondary: 05B35, 05C25, 05C65, 68R10, 11D0

Key words and phrases. Diophantine equations, Hyperplane, Grassmannian, Radon transform of discrete matrices, Inversion formula, Support theorem.

\section{Introduction.}

In the literature of the notion of integral geometry we find several authors who had approached this field, we cite among others: Gelfand [1], Gonzalez [2], Helgason [3] and Rubin [4]. Their contributions consisted in studying the Radon transform on the Grassmannian manifolds and projective spaces. The purpose of this paper is to study the Radon transform $R$ on the Grassmannian $\mathbb{G}$ of the ring of discrete matrices $\mathcal{M}_{n}(\mathbb{Z}), \mathbb{G}$ is the set of hyperplanes of $\mathcal{M}_{n}(\mathbb{Z})$ These hyperplanes are defined in a natural way by means of the linear form trace of $\mathcal{M}_{n}(\mathbb{Z})$. By analogy with the integral geometry in the euclidean case, a specific properties of the $R$ and its dual $R^{\star}$, inversion formula and support theorem for this transform are treated.

Throughout the following $n$ denotes an integer $\geq 2$ and $\mathcal{M}_{n}(\mathbb{Z})$ the ring of square matrices of size $n$ with coefficients in $\mathbb{Z}$. All the multiplications are in the matrices sense. A single

Received : December 02, 2020 - Accepted: March 10, 2021.

(C) The Author(s) 2021. This article is published with open access by Sidi Mohamed Ben Abdallah University.

${ }^{1}$ Hassan II University, Higher Normal School Casablanca, Morocco.

e-mail: abdelbaki.attioui@gmail.com. 
column or single row matrix will be noted in the same way as a vector. For a given $a \in \mathcal{M}_{n}(\mathbb{Z})$ and $b \in \mathbb{Z}$, we consider the equation,

$$
\operatorname{Tr}(a x)=b, \quad x \in \mathcal{M}_{n}(\mathbb{Z})
$$

where $\operatorname{Tr}$ is the operator trace of $\mathcal{M}_{n}(\mathbb{Z})$. In other words,

$$
\sum_{i=1}^{n} a_{i .} x_{. i}=b
$$

where $a_{i .}$ denotes the ith row of the matrix $a=\left(a_{i j}\right)$ and $x_{. i}$ the ith column of the matrix $x=\left(x_{i j}\right)$.

Therefore, solving equation (1.1) in $\mathcal{M}_{n}(\mathbb{Z})$ amounts to solving linear Diophantine equation (1.2) in $\mathbb{Z}^{n^{2}}$. In the following paragraph, we give a description of the solutions of such equation using the normal Hermite form. Then, we give a necessary and sufficient condition to have an infinity of solutions of equation (1.1).

1.1. Linear Diophantine equation. Let $m$ an integer $\geq 2$, for $a \in \mathbb{Z}^{m}$ and $b \in \mathbb{Z}$, the set of solutions in $\mathbb{Z}^{m}$ of the linear Diophantine equation $a x=b$, is denote by

$$
E_{m}(a, b)=\left\{x \in \mathbb{Z}^{m}: a x=b\right\}
$$

It is well known that a necessary condition of existence the solutions is $d(a)$ divide $b$, where $d(a)$ designates the greatest common divisor of the integers components of $a$.

Lemma 1.1. Let $a \in \mathbb{Z}^{m}, a \neq 0$. Then, there exists a matrix $R \in G L_{m}(\mathbb{Z})$ such that the (m-1) last columns of $R$ form a basis of $E_{m}(a, 0)$ as a $\mathbb{Z}$-submodule of $\mathbb{Z}^{m}$.

Proof. It is clear $E_{m}(a, 0)$ is a $\mathbb{Z}$-submodule of $\mathbb{Z}^{m}$. Because we have $E_{m}(a, 0)=E_{m}\left(\frac{a}{d(a)}, 0\right)$ and $d\left(\frac{a}{d(a)}\right)=1$, we can suppose $d(a)=1$. For $m=2$, by Bezout theorem, there exists $u_{1}, u_{2} \in \mathbb{Z}$ : $a_{1} u_{1}+a_{2} u_{2}=1$, then

$$
R=\left(\begin{array}{cc}
u_{1} & -a_{2} \\
u_{2} & a_{1}
\end{array}\right) \in G L_{2}(\mathbb{Z}) \text { and } a R=\left(a_{1}, a_{2}\right)\left(\begin{array}{cc}
u_{1} & -a_{2} \\
u_{2} & a_{1}
\end{array}\right)=(1,0)
$$

But, $(1,0)\left(y_{1}, y_{2}\right)=0$ if and only if $y_{1}=0$ and $y_{2}=t$, any $t \in \mathbb{Z}$. Thus,

$$
a x=0 \Longleftrightarrow\left(x_{1}, x_{2}\right)=R(0, t)=\left(-t a_{2}, t a_{1}\right), t \in \mathbb{Z}
$$

then,

$$
E_{2}(a, 0)=\left\{t\left(-a_{2}, a_{1}\right): t \in \mathbb{Z}\right\}=\mathbb{Z} R_{2} \text {, with } R_{2} \text { is 2nd column of } R
$$

We can apply the above to vectors of length $m>2$. By elementary operations on the matrix $a$, we come back to a matrix of the form $(1,0, \ldots, 0)$. Let $R$ the matrix of elementary formatting operations staggered. We therefore have $a R=(1,0, \ldots, 0)$. By using this, one easily obtains,

for all $a \in \mathbb{Z}^{m}: d(a)=1$, there exists $R \in G L_{m}(\mathbb{Z}): a R=(1,0 \ldots, 0)$.

Put, $R_{j}=\left(u_{1, j}, \ldots, u_{m, j}\right)$ the $j$ th column of $R, j \in\{1, \ldots, m\}$. Let $x \in E_{m}(a, 0)$ and $y=R^{-1} x$, then

$$
a x=a R R^{-1} x=(1,0, \ldots, 0) y=0 \Longleftrightarrow y=\left(0, t_{2}, \ldots, t_{m}\right), \quad t_{2}, \ldots, t_{m} \in \mathbb{Z}
$$


so,

$$
x=R\left(0, t_{2}, \ldots, t_{m}\right)=\left(\sum_{j=2}^{m} t_{j} u_{1, j}, \ldots, \sum_{j=2}^{m} t_{j} u_{m, j}\right)=\sum_{k=2}^{m} t_{k} R_{k}
$$

Finally,

$$
E_{m}(a, 0)=\left\{\sum_{j=2}^{m} t_{j} R_{j}: t_{2}, \ldots, t_{m} \in \mathbb{Z}\right\}
$$

Example 1.1. Let $a \in \mathbb{Z}^{m}$ such that $a_{1} \neq 0, d\left(a_{1}, a_{2}\right)=1$, then $d(a)=1, a_{1} u_{1,1}+a_{2} u_{2,1}=1$ where $u_{1,1}, u_{2,1} \in \mathbb{Z}$ and the columns of such a matrix $\mathrm{R}$ are given by

$$
\left\{\begin{array}{l}
R_{1}=\left(u_{1,1}, u_{2,1}, 0, \ldots, 0\right) \\
R_{2}=\left(-a_{2}, a_{1}, 0, \ldots, 0\right) \\
R_{j}=(-a_{j} u_{1,1},-a_{j} u_{2,1}, 0 \ldots, 0, \underbrace{1}_{j \text { th }}, 0, \ldots, 0), \text { for } j=3, \ldots, m
\end{array}\right.
$$

We have $R \in G L_{m}(\mathbb{Z})$, $\operatorname{det}(R)=1$ and $a R=(1,0 \ldots, 0)$.

Theorem 1.1. Let $m$ an integer $\geq 2, a \in \mathbb{Z}^{m}$. Then, $E_{m}(a, b)$ is an infinite subset of $\mathbb{Z}^{m}$, for all $b \in \mathbb{Z}$, if and only if $d(a)=1$. In this case we have

$$
E_{m}(a, b)=b R_{1}+\mathbb{Z} R_{2}+\ldots+\mathbb{Z} R_{m}
$$

where $R_{k}$ are the columns of $R \in G L_{m}(\mathbb{Z})$ such that $a R=(1,0 \ldots, 0)$.

Proof. The sufficient condition is easily deduced from the lemma 1.1, moreover we have equality (1.3). Conversely, we assume $E_{m}(a, b)$ is an infinite set, for all $b \in \mathbb{Z}$. In particular, the set $E_{m}(a, 1)$ is not empty, by Bezout theorem, $d(a)=1$.

Theorem 1.2. Let $m$ an integer $\geq 2, a, a^{\prime} \in \mathbb{Z}^{m}: d(a)=d\left(a^{\prime}\right)=1$ and $b \in \mathbb{Z}, b \neq 0$. Then, $E_{m}(a, b)=E_{m}\left(a^{\prime}, b\right)$ if and only if $a^{\prime}=a$.

Proof. Just the necessary condition to prove. Theorem 1.1 gives $R, R^{\prime} \in G L_{m}(\mathbb{Z})$ such that $a R=a^{\prime} R^{\prime}=(1,0 \ldots, 0)$ and

$$
\begin{aligned}
& E_{m}(a, b)=\left\{b R_{1}+\sum_{j=2}^{m} t_{j} R_{j}: t_{2}, \ldots, t_{m} \in \mathbb{Z}\right\} \\
& E_{m}\left(a^{\prime}, b\right)=\left\{b R_{1}^{\prime}+\sum_{j=2}^{m} t_{j} R_{j}^{\prime}: t_{2}, \ldots, t_{m} \in \mathbb{Z}\right\}
\end{aligned}
$$

where $R_{j}$ and $R_{j}^{\prime}$ are respectively the jth column of $R$ and $R^{\prime}$. By hypothesis, for all $t_{2}, \ldots, t_{m} \in$ $\mathbb{Z}$ and $t_{2}^{\prime}, \ldots, t_{m}^{\prime} \in \mathbb{Z}$,

$$
\left\{\begin{array}{l}
a\left(b R_{1}^{\prime}+\sum_{j=2}^{m} t_{j} R_{j}^{\prime}\right)=b \\
a^{\prime}\left(b R_{1}+\sum_{j=2}^{m} t_{j}^{\prime} R_{j}\right)=b
\end{array}\right.
$$


then,

$$
\left\{\begin{array}{l}
b a^{\prime} R_{1}=b a R_{1}^{\prime}=b \\
a R_{j}^{\prime}=a^{\prime} R_{j}=0, \text { for all } j=2, \ldots, m
\end{array}\right.
$$

Since, $b \neq 0$,

$$
a^{\prime} R_{1}=a R_{1}^{\prime}=1 \text { and } a^{\prime} R=a R^{\prime}=(1,0, \ldots, 0) .
$$

We have $a R=a^{\prime} R^{\prime}=(1,0, \ldots, 0)$, then $\left(a^{\prime}-a\right) R=(0, \ldots, 0)$. This gives $a^{\prime}=a$, because $R$ is an invertible matrix.

Theorem 1.3. Let $m$ an integer $\geq 2, a, a^{\prime} \in \mathbb{Z}^{m}: d(a)=d\left(a^{\prime}\right)=1$. Then, $E_{m}(a, 0)=E_{m}\left(a^{\prime}, 0\right)$ if and only if $a^{\prime}=\varepsilon a$, with $\varepsilon= \pm 1$.

Proof. For $1 \leq i, j \leq m$ with $i \neq j$, let $x=\left(x_{1}, \ldots, x_{m}\right) \in \mathbb{Z}^{m}$ defined by

$$
x_{k}= \begin{cases}-a_{j} & \text { if } k=i \\ a_{i} & \text { if } k=j \\ 0 & \text { elsewhere }\end{cases}
$$

We have $x \in E_{m}(a, 0)$, then $x \in E_{m}\left(a^{\prime}, 0\right)$ i.e. $a^{\prime} x=0$. It follows

$$
a_{i}^{\prime} a_{j}=a_{j}^{\prime} a_{i}, \quad \text { for all } i, j \in\{1, \ldots, m\}, i \neq j
$$

On the one hand, $a_{i}=0$ if and only if $a_{i}^{\prime}=0$, because $d(a)=d\left(a^{\prime}\right)=1, a_{j}$ and $a_{j}^{\prime}$ are not all zero. Then,

$$
I:=\left\{i \in\{1, \ldots, m\}: a_{i} \neq 0\right\}=\left\{i \in\{1, \ldots, m\}: a_{i}^{\prime} \neq 0\right\}
$$

Suppose that $I$ contains at least two elements. Otherwise $I=\{i\}, a_{i}= \pm 1, a_{i}^{\prime}= \pm 1$, because $d(a)=d\left(a^{\prime}\right)=1$, and in this case $a= \pm a^{\prime}$. On the other hand, let us set $u=\left(u_{1}, \ldots, u_{m}\right) \in \mathbb{Z}^{m}$ the Bezout coefficients associated with $a$. We have, for all $i \in I$,

$$
a_{i}^{\prime} \sum_{j \in I, j \neq i} a_{j} u_{j}=\sum_{j \in I, j \neq i} a_{i}^{\prime} a_{j} u_{j}=\sum_{j \in I, j \neq i} a_{j}^{\prime} a_{i} u_{j}=a_{i} \sum_{j \in I, j \neq i} a_{j}^{\prime} u_{j}
$$

But, $a u=\sum_{j \in I} a_{j} u_{j}=1$ and $a^{\prime} u=\sum_{j \in I} a_{j}^{\prime} u_{j}$, then

$$
a_{i}^{\prime}\left(1-a_{i} u_{i}\right)=a_{i}\left(a^{\prime} u-a_{i}^{\prime} u_{i}\right) \Longleftrightarrow a_{i}^{\prime}=a_{i} a^{\prime} u
$$

In the same way we show $a_{i}=a_{i}^{\prime} a u^{\prime}$. Hence, for all $i \in I, a_{i}^{\prime}=\varepsilon_{i} a_{i}$, with $\varepsilon_{i}= \pm 1$. Then, by (1.4),

$$
\varepsilon_{i} a_{i} a_{j}=\varepsilon_{j} a_{j} a_{i}, \quad \text { for all } i, j \in I, i \neq j
$$

that is, $\varepsilon_{i}=\varepsilon_{j}$, for all $i, j \in I$, then

$$
a_{i}^{\prime}=\varepsilon a_{i}, \quad \text { for all } i \in I, \text { with } \varepsilon= \pm 1
$$

Finally, $a^{\prime}=\varepsilon a$. 
1.2. Hyperplanes and Grassmannian of $\mathcal{M}_{n}(\mathbb{Z})$. Let $\mathcal{P}=\left\{a \in \mathcal{M}_{n}(\mathbb{Z}): d(a)=1\right\}$. By theorem 1.4 below, $\mathcal{P}$ is the set of all matrices $a \in \mathcal{M}_{n}(\mathbb{Z})$ such that the equation (1.1) admits infinity of solutions in $\mathcal{M}_{n}(\mathbb{Z})$. In a way $\mathcal{P}$ plays the role of the sphere in the Euclidean case.

A discrete hyperplane of $\mathcal{M}_{n}(\mathbb{Z})$ is defined as

$$
H(a, b)=\left\{x \in \mathcal{M}_{n}(\mathbb{Z}): \operatorname{Tr}(a x)=b\right\}, \quad a \in \mathcal{P}, b \in \mathbb{Z}
$$

The Grassmannian of $\mathcal{M}_{n}(\mathbb{Z})$ is defined as

$$
\mathbb{G}=\{H(a, b): a \in \mathcal{P}, b \in \mathbb{Z}\}
$$

Theorem 1.4. Let $a \in \mathcal{M}_{n}(\mathbb{Z})$. Then, $H(a, b)$ is an infinite set, for all $b \in \mathbb{Z}$ if and only if $a \in \mathcal{P}$. Proof. Looking at $a=\left(a_{i j}\right) \in \mathcal{M}_{n}(\mathbb{Z})$ as a vector $a=\left(a_{1 .}, \ldots, a_{n}\right) \in \mathbb{Z}^{n^{2}}$, where $a_{i}$. is the ith row of the matrix $a$, we have

$$
H(a, b)=\left\{x \in \mathcal{M}_{n}(\mathbb{Z}):\left(x_{1 .}, \ldots, x_{n .}\right) \in E_{n^{2}}(a, b)\right\},
$$

If $d(a)=1$, by theorem 1.1, we have $H(a, b)$ is an infinite set, for all $b \in \mathbb{Z}$. Reciprocally, assume $H(a, b)$ is an infinite set for all $b \in \mathbb{Z}$. In particular, for $b=1, E_{n^{2}}(a, 1)$ is not empty then $d(a)=1$, by Bezout theorem.

Theorem 1.5. Let $a, a^{\prime} \in \mathcal{P}$ and $b, b^{\prime} \in \mathbb{Z}$. Then, $H(a, b)=H\left(a^{\prime}, b^{\prime}\right)$ if and only if

$$
a^{\prime}=\varepsilon a \text { and } b^{\prime}=\varepsilon b \text {, where } \varepsilon= \pm 1
$$

Proof. It is obvious that if $a^{\prime}=\varepsilon a$ and $b^{\prime}=\varepsilon b$, where $\varepsilon= \pm 1$, then $H(a, b)=H\left(a^{\prime}, b^{\prime}\right)$. We just prove the other direction.

Suppose that $H(a, b)=H\left(a^{\prime}, b^{\prime}\right)$. Thus, the Diophantine equations

$$
\left\{\begin{array}{l}
\operatorname{Tr}(a x)=b \\
\operatorname{Tr}\left(a^{\prime} x\right)=b^{\prime}
\end{array}\right.
$$

have the same solutions in $\mathcal{M}_{n}(\mathbb{Z})$.

Since $d(a)=d\left(a^{\prime}\right)=1$, by Bezout theorem, there exists $y, y^{\prime} \in \mathcal{M}_{n}(\mathbb{Z})$ such that $\operatorname{Tr}(a y)=$ $\operatorname{Tr}\left(a^{\prime} y^{\prime}\right)=1$. Then, $b y, b^{\prime} y^{\prime} \in H(a, b)=H\left(a^{\prime}, b^{\prime}\right)$. Therefore, $\left\{\begin{array}{l}b^{\prime} \operatorname{Tr}\left(a y^{\prime}\right)=\operatorname{Tr}\left(a\left(b^{\prime} y^{\prime}\right)\right)=b \\ b \operatorname{Tr}\left(a^{\prime} y\right)=\operatorname{Tr}\left(a^{\prime}(b y)\right)=b^{\prime}\end{array}\right.$. Hence, $b^{\prime}=\varepsilon b$ with $\varepsilon= \pm 1$. Consequently,

$$
H(a, b)=H\left(a^{\prime}, b^{\prime}\right)=H\left(a^{\prime}, \varepsilon b\right)=H\left(\varepsilon a^{\prime}, b\right)
$$

This means

$$
E_{n^{2}}(a, b)=E_{n^{2}}\left(\varepsilon a^{\prime}, b\right)
$$

If $b \neq 0$, since $d\left(\varepsilon a^{\prime}\right)=d\left(a^{\prime}\right)=d(a)=1$, by theorem $1.2, a^{\prime}=\varepsilon^{\prime} \varepsilon a$, with $\varepsilon^{\prime}= \pm 1$. But,

$$
\operatorname{Tr}(a x)=b \Longleftrightarrow \operatorname{Tr}\left(a^{\prime} x\right)=b^{\prime} \Longleftrightarrow \operatorname{Tr}\left(\varepsilon^{\prime} \varepsilon a x\right)=\varepsilon b \Longleftrightarrow \varepsilon^{\prime} \operatorname{Tr}(a x)=b
$$

then, $\varepsilon^{\prime}=1$ and $a^{\prime}=\varepsilon a$. If $b=0$, then $b^{\prime}=0$ and by theorem $1.3, a^{\prime}=\varepsilon a$.

Proposition 1.1. Let $a \in \mathcal{P}$. Then the $\mathbb{Z}$-module $\mathcal{M}_{n}(\mathbb{Z})$ can be written in the form, disjoint union,

$$
\mathcal{M}_{n}(\mathbb{Z})=\bigsqcup_{b \in \mathbb{Z}} H(a, b)
$$


Proof. Let $a \in \mathcal{P}$ and $x \in \mathcal{M}_{n}(\mathbb{Z})$. By taking $b=\operatorname{Tr}(a x) \in \mathbb{Z}$, it is clear that $x$ belongs to the discrete hyperplane $H(a, b)$. Then $\mathcal{M}_{n}(\mathbb{Z})$ is contained in $\bigsqcup_{b \in \mathbb{Z}} H(a, b)$, and therefore $\mathcal{M}_{n}(\mathbb{Z})=\bigsqcup_{b \in \mathbb{Z}} H(a, b)$.

It is clear that $H(a, b) \cap H\left(a, b^{\prime}\right)=\varnothing$ as soon as $b \neq b^{\prime}$. This complete the proof of proposition.

\section{Radon transform on $\mathcal{M}_{n}(\mathbb{Z})$}

To define the Radon transform on the Grassmanian of $\mathcal{M}_{n}(\mathbb{Z})$, we need the following fundamental functional spaces.

2.1. Functional spaces for Radon transform on $\mathcal{M}_{n}(\mathbb{Z})$. Let

$$
\ell^{1}\left(\mathcal{M}_{n}(\mathbb{Z})\right)=\left\{f: \mathcal{M}_{n}(\mathbb{Z}) \rightarrow \mathbb{C}:\|f\|_{1}:=\sum_{x \in \mathcal{M}_{n}(\mathbb{Z})}|f(x)|<\infty\right\}
$$

and

$$
\mathcal{S}\left(\mathcal{M}_{n}(\mathbb{Z})\right)=\left\{f \in l^{1}\left(\mathcal{M}_{n}(\mathbb{Z})\right): p_{N}(f)<+\infty, \text { for all } N \in \mathbb{N}\right\}
$$

where $p_{N}(f)=\sup _{x \in \mathcal{M}_{n}(\mathbb{Z})}\left(1+\|x\|_{F}^{2}\right)^{N}|f(x)|$ and $\|x\|_{F}$ is the Frobenius norm of the matrix $x$.

We note that $\mathcal{S}\left(\mathcal{M}_{n}(\mathbb{Z})\right)$ is a remarkable subspace of $\ell^{1}\left(\mathcal{M}_{n}(\mathbb{Z})\right)$ equipped with the family of semi-norms $\left(p_{N}\right)_{N \in \mathbb{N}}$ is a metrisable locally convex topological vector space and is called the Schwartz space of $\mathcal{M}_{n}(\mathbb{Z})$.

Let $\mathbb{C}^{\left(\mathcal{M}_{n}(\mathbb{Z})\right)}$ be the subspace of $\mathcal{S}\left(\mathcal{M}_{n}(\mathbb{Z})\right)$ consisting of all complex-valued functions $f$ defined on $\mathcal{M}_{n}(\mathbb{Z})$ with finite support. It is well known that $\mathbb{C}^{\left(\mathcal{M}_{n}(\mathbb{Z})\right)}$ is dense in $\mathcal{S}\left(\mathcal{M}_{n}(\mathbb{Z})\right)$.

Recall that $\mathbb{G}=\{H(a, b): a \in \mathcal{P}, b \in \mathbb{Z}\}$. For $a \in \mathcal{P}$ and $b \in \mathbb{Z}$, we denote $\mathbb{G}(a,$.$) and \mathbb{G}(., b)$, the subsets of $G$,

$$
\mathbb{G}(a, .)=\{H(a, z): z \in \mathbb{Z}\}, \quad \mathbb{G}(., b)=\{H(x, b): x \in \mathcal{P}\}
$$

and for $x \in \mathcal{M}_{n}(\mathbb{Z})$,

$$
\mathbb{G}_{x}=\{g \in \mathbb{G}: x \in g\}
$$

Let $\ell^{1}(\mathbb{G})$ the space of all complex-valued functions $F$ defined on $\mathbb{G}$ such that

$$
\sum_{g \in \mathbb{G}(a, .)}|F(g)|<+\infty, \text { for all } a \in \mathcal{P}
$$

A Schwartz space type of $\mathbb{G}$, denoted by $\mathcal{S}_{*}(\mathbb{G})$, is the subspace of $\ell^{1}(\mathbb{G})$ consisting of all functions $F \in \ell^{1}(\mathbb{G})$ such that $q_{N}(F)<+\infty$ for all $N \in \mathbb{N}$. For $N \in \mathbb{N}$, the semi norm $q_{N}$ is defined by

$$
q_{N}(F)=\sup _{g \in \mathbb{G}}\left(1+|g|^{2}\right)^{N}|F(g)|, \text { for all } F \in \mathcal{S}_{*}(\mathbb{G}),
$$

where, for $g=H(a, b),|g|^{2}=\left.|| a\right|_{F} ^{2}+b^{2}$ is well defined by Theorem1.5. 
Remark 2.1. If $F \in \mathcal{S}_{*}(\mathbb{G})$, then the series $\sum_{g \in \mathbb{G}} F(g)$ converge absolutely. Indeed, by (2.1) for a integer $N>1$ and for all $g=H(a, b) \in \mathbb{G}$

$$
|F(g)| \leq q_{2 N}(F) \frac{1}{\left(1+\|a\|_{F}^{2}\right)^{N}} \frac{1}{\left(1+b^{2}\right)^{N}}
$$

Then, for $2 N>n^{2}$,

$$
\sum_{g \in \mathbb{G}}|F(g)| \leq q_{2 N}(F) \sum_{a \in \mathcal{P}} \frac{1}{\left(1+\|a\|_{F}^{2}\right)^{N}} \sum_{b \in \mathbb{Z}} \frac{1}{\left(1+b^{2}\right)^{N}}<\infty
$$

\subsection{Radon transform and its dual on $\mathcal{M}_{n}(\mathbb{Z})$.}

Definition 2.1. Let $f \in \ell^{1}\left(\mathcal{M}_{n}(\mathbb{Z})\right)$. The discrete Radon transform of $f$ is the complex-valued function $R f$ defined on $G$ by

$$
R f(g)=\sum_{x \in g} f(x), \text { for all } g \in \mathbb{G}
$$

Definition 2.2. Let $F \in \mathcal{S}_{*}(\mathbb{G})$. The dual discrete transform of $F$ is the complex-valued function $R^{*} F$ defined on $\mathcal{M}_{n}(\mathbb{Z})$ by

$$
R^{*} F(x)=\sum_{g \in \mathbb{G}_{x}} F(g), \text { for all } x \in \mathcal{M}_{n}(\mathbb{Z}),
$$

Note that the series is absolutely convergent, according to the remark 2.1.

As in the Euclidean case, we now show the invariance of the discrete Radon transform under translations. More precisely, we have

Proposition 2.1. Let $f \in \ell^{1}\left(\mathcal{M}_{n}(\mathbb{Z})\right)$ and $\left(x_{0}, b_{0}\right) \in \mathcal{M}_{n}(\mathbb{Z}) \times \mathbb{Z}$. Then, for all $(a, b) \in \mathcal{P} \times \mathbb{Z}$ such that $x_{0} \in H\left(a, b_{0}\right)$, the following equality holds

$$
R\left(x_{0} f\right)(H(a, b))=R f\left(H\left(a, b+b_{0}\right)\right),
$$

where $\left(x_{0} f\right)(x)=f\left(x+x_{0}\right)$, for all $x \in \mathcal{M}_{n}(\mathbb{Z})$.

Proof. By definition (2.3), we have

$$
R\left(x_{0} f\right)(H(a, b))=\sum_{x \in H(a, b)} f\left(x+x_{0}\right)=\sum_{y \in x_{0}+H(a, b)} f(y)
$$

but

$$
y \in x_{0}+H(a, b) \Longleftrightarrow \operatorname{Tr}(a y)=b+\operatorname{Tr}\left(a x_{0}\right)=b+b_{0} \Longleftrightarrow y \in H\left(a, b+b_{0}\right)
$$

then,

$$
R\left(x_{0} f\right)(H(a, b))=\sum_{y \in H\left(a, b+b_{0}\right)} f(y)=R f\left(H\left(a, b+b_{0}\right)\right) .
$$


Proposition 2.2 (Duality formula). Let $f \in \ell^{1}\left(\mathcal{M}_{n}(\mathbb{Z})\right)$ and $F \in \mathcal{S}_{*}(\mathbb{G})$. Then

$$
\sum_{x \in \mathcal{M}_{n}(\mathbb{Z})} f(x) R^{\star} F(x)=\sum_{g \in \mathbb{G}} R f(g) F(g)
$$

Proof. Note that the series above are absolutely convergent see Remark 2.1. Then, we can write

$$
\begin{aligned}
\sum_{x \in \mathcal{M}_{n}(\mathbb{Z})} f(x) R^{\star} F(x) & =\sum_{x \in \mathcal{M}_{n}(\mathbb{Z})} f(x) \sum_{g \in \mathbb{G}_{x}} F(g) \\
& =\sum_{x \in \mathcal{M}_{n}(\mathbb{Z})} f(x) \sum_{g \in \mathbb{G}}\left(F \mathbf{1}_{\mathbb{G}_{x}}\right)(g) \\
& =\sum_{g \in \mathbb{G}} F(g) \sum_{x \in \mathcal{M}_{n}(\mathbb{Z})} f(x) \mathbf{1}_{\mathbb{G}_{x}}(g) \\
& =\sum_{g \in \mathbb{G}} F(g) \sum_{x \in g} f(x) \\
& =\sum_{g \in \mathbb{G}} R f(g) F(g)
\end{aligned}
$$

where $\mathbf{1}_{A}$ denotes the characteristic function of a set $A$.

In following, we extend the inversion formula established in [2] to invert the Radon transform $R$ of discrete matrices.

Theorem 2.1 (Inversion Theorem). Let $f \in \ell^{1}\left(\mathcal{M}_{n}(\mathbb{Z})\right)$ and $x_{0} \in \mathcal{M}_{n}(\mathbb{Z})$. Then, there exists a sequence $\left(g_{k}\right)_{k \geq 1}$ of $\mathbf{G}_{x_{0}}$ such that

$$
f\left(x_{0}\right)=\lim _{k \rightarrow+\infty} R f\left(g_{k}\right)
$$

Proof. By proposition 2.1, we can assume $x_{0}=0$. It is a question of constructing a sequence $\left(g_{k}\right)_{k \geq 1}$ of $\mathbf{G}_{0}$ such that

$$
f(0)=\lim _{k \rightarrow+\infty} \sum_{x \in g_{k}} f(x)
$$

But the hyperplane $g_{k} \in \mathbb{G}_{0}$ is written as $g_{k}=H\left(a_{k}, 0\right)$ with $a_{k} \in \mathcal{P}$. For an integer $k \geq 1$, we consider the matrix $a_{k}=\left(\left(a_{k}\right)_{i, j}\right)_{1 \leq i, j \leq n}$ of $\mathcal{M}_{n}(\mathbb{Z})$ defined by:

$$
a_{k}=\left(\begin{array}{cccc}
1 & k & \ldots & k^{n-1} \\
k^{n} & k^{n+1} & \ldots & k^{2 n-1} \\
\vdots & \vdots & \vdots & \vdots \\
k^{n^{2}-n} & k^{n^{2}-n+1} & \ldots & k^{n^{2}-1}
\end{array}\right)=\left(k^{(i-1) n+j-1}\right)_{1 \leq i, j \leq n}
$$

Let $x=\left(x_{i, j}\right)_{1 \leq i, j \leq n} \in \mathcal{M}_{n}(\mathbb{Z})$. For $1 \leq i, j \leq n$, put $x_{(j-1) n+i-1}:=x_{i, j}$, we have

$$
x=\left(x_{i, j}\right) \in H\left(a_{k}, 0\right) \Longleftrightarrow \operatorname{Tr}\left(a_{k} x\right)=\sum_{i=1}^{n} \sum_{j=1}^{n} k^{(i-1) n+j-1} x_{j, i}=0
$$

or

$$
x \in g_{k} \Longleftrightarrow \sum_{p=0}^{n^{2}-1} k^{p} x_{p}=\sum_{i=1}^{n} \sum_{j=1}^{n} k^{(i-1) n+j-1} x_{(i-1) n+j-1}=0
$$


Otherwise,

$$
\|x\|_{F}^{2}=\sum_{i=1}^{n} \sum_{j=1}^{n} x_{i, j}^{2}=\sum_{p=0}^{n^{2}-1} x_{p}^{2}
$$

Let $x \in g_{k}$ with $x \neq 0$, then we have $\|x\|_{F} \geq k$. Indeed, let

$$
p_{0}=\min \left\{0 \leq p \leq n^{2}-1: x_{p} \neq 0\right\}
$$

If $p_{0}=0$, then

$$
x_{p_{0}}=-\sum_{p=1}^{n^{2}-1} k^{p} x_{p}=-k \sum_{p=1}^{n^{2}-1} k^{p-1} x_{p}
$$

Consequently, $k$ divides $x_{p_{0}} \neq 0$, hence $x_{p_{0}}^{2} \geq k^{2}$.

If $p_{0} \geq 1$, then, $x_{0}=\ldots=x_{p_{0}-1}=0$, and

$$
k^{p_{0}} x_{p_{0}}=-\sum_{p=p_{0}+1}^{n^{2}-1} k^{p} x_{p}=-k^{p_{0}+1} \sum_{p=p_{0}+1}^{n^{2}-1} k^{p-p_{0}-1} x_{p}
$$

It follows, $k$ divides $x_{p_{0}} \neq 0$, hence $x_{p_{0}}^{2} \geq k^{2}$. Thus,

$$
\|x\|_{F}^{2} \geq x_{p_{0}}^{2} \geq k^{2}
$$

We have then

$$
\forall x \in g_{k}, \quad x \neq 0 \Longleftrightarrow\|x\|_{F} \geq k .
$$

We deduce that, for $f \in \ell^{1}\left(\mathcal{M}_{n}(\mathbb{Z})\right)$,

$$
R f\left(g_{k}\right)=\sum_{x \in g_{k}} f(x)=f(0)+\sum_{\|x\|_{F} \geq k} f(x)
$$

Since $f \in \ell^{1}\left(\mathcal{M}_{n}(\mathbb{Z})\right), \lim _{k \rightarrow \infty} \sum_{\|x\|_{F} \geq k} f(x)=0$. So, $\lim _{k \rightarrow \infty} R f\left(g_{k}\right)=f(0)$.

Now, we will state support theorem by extending the support theorem stated in [2]. We shall study the support of a function $f \in \mathbb{C}^{\left(\mathcal{M}_{n}(\mathbb{Z})\right)}$ as well as the support of $R f$. We begin with the following useful lemmas. We designates $\mathbf{1}_{A}$ the characteristic function of a set $A$.

Lemma 2.1. Let $x_{0} \in \mathcal{M}_{n}(\mathbb{Z})$. Then $R \mathbf{1}_{\left\{x_{0}\right\}}=\mathbf{1}_{\mathrm{G}_{x_{0}}}$.

Proof. Let $x_{0} \in \mathcal{M}_{n}(\mathbb{Z})$ and $g \in \mathbb{G}$ we have

$$
R \mathbf{1}_{\left\{x_{0}\right\}}(g)=\sum_{x \in g} \mathbf{1}_{\left\{x_{0}\right\}}(x)=\left\{\begin{array}{ll}
1 & \text { if } x_{0} \in g \\
0 & \text { otherwise }
\end{array}=\mathbf{1}_{\mathbb{G}_{x_{0}}}(g) .\right.
$$

The lemma is proved.

Lemma 2.2. Let $x_{0} \in \mathcal{M}_{n}(\mathbb{Z})$. Then Supp $R \mathbf{1}_{\left\{x_{0}\right\}}=\mathbb{G}_{x_{0}}$. 
Proof. Let $x_{0} \in \mathcal{M}_{n}(\mathbb{Z})$. From lemma 2.1, we have $R \mathbf{1}_{\left\{x_{0}\right\}}=\mathbf{1}_{\mathbb{G}_{x_{0}}}$. Since, $g \in \mathbb{G}_{x_{0}} \Longleftrightarrow x_{0} \in g$, then Supp $\mathbf{1}_{\mathbb{G}_{x_{0}}}=\mathbb{G}_{x_{0}}$. Therefore,

$$
\operatorname{Supp} R \mathbf{1}_{\left\{x_{0}\right\}}=\operatorname{Supp} \mathbf{1}_{\mathbb{G}_{x_{0}}}=\mathbb{G}_{x_{0}}
$$

Theorem 2.2 (Support Theorem). Let $f \in \mathbb{C}^{\left(\mathcal{M}_{n}(\mathbb{Z})\right)}$ and $K=\left\{x_{1}, \ldots, x_{r}\right\}$ a subset of $\mathcal{M}_{n}(\mathbb{Z})$, with $r$ an integer $\geq 1$. Then

$$
\operatorname{Supp}(f) \subset K \Longleftrightarrow \operatorname{Supp}(R f) \subset \bigcup_{i=1}^{r} \mathbb{G}_{x_{i}}
$$

Proof. Suppose $\operatorname{Supp}(f) \subset K$, then $f=\sum_{i=1}^{r} f\left(x_{i}\right) \mathbf{1}_{\left\{x_{i}\right\}}$. By lemma 2.1,

$$
R f=\sum_{i=1}^{r} f\left(x_{i}\right) \mathbf{1}_{\mathrm{G}_{x_{i}}}
$$

Let $g \notin \bigcup_{i=1}^{r} \mathbb{G}_{x_{i}}$, by definition $x_{i} \notin g$, for all $i=1, \ldots, r$. In the other words, $\mathbf{1}_{\mathrm{G}_{x_{i}}}(g)=0$, for all $i=1, \ldots, r$. So by expression (2.9), $R f(g)=0$. It follows that

$$
\operatorname{Supp}(R f) \subset \bigcup_{i=1}^{r} \mathbb{G}_{x_{i}} \text {. }
$$

Conversely, suppose that we have the inclusion (2.10). Let $x_{0} \notin K$, by inversion Theorem,

$$
f\left(x_{0}\right)=\lim _{k \rightarrow+\infty} R f\left(g_{k}\right)
$$

where $\left(g_{k}\right)_{k \geq 1}$ is a sequence of $\mathbb{G}_{x_{0}}$. That is $x_{0} \in g_{k}$, for all $k \geq 1$. To prove $f\left(x_{0}\right)=0$, it suffices to prove that $R f\left(g_{k}\right)=0$ is zero from a certain rank. By assumption (2.10), it suffices to prove that there exists $k_{0} \in \mathbb{N}$ such that $g_{k} \notin \bigcup_{i=1}^{r} G_{x_{i}}$, for all $k \geq k_{0}$. By construction, $g_{k}=H\left(a_{k}, a_{k} x_{0}\right)$, where the matrix $a_{k}$ is given by formula (2.6). It is then a question of showing that there exists $k_{0} \in \mathbb{N}$ such that

$$
x_{i}-x_{0} \notin H\left(a_{k}, 0\right) \text {, for all } i=1, \ldots, r \text { and for all } k \geq k_{0}
$$

Let

$$
k_{0}=\max _{1 \leq i \leq r}\left\|x_{i}-x_{0}\right\|_{F}^{2}+1,
$$

then $k_{0} \in \mathbb{N}$ and for all $k \geq k_{0}$, and for all $i \in\{1, \ldots, r\}$

$$
0<\left\|x_{i}-x_{0}\right\|_{F}<k_{0} \leq k
$$

From (2.7) it follows that, $x_{i}-x_{0} \notin H\left(a_{k}, 0\right)$ or equivalently $x_{i} \notin g_{k}$. The theorem is proved

Remark 2.2. Let $f \in \mathbb{C}^{\left(\mathcal{M}_{n}(\mathbb{Z})\right)}$ and $K \subset \mathcal{M}_{n}(\mathbb{Z})$ a finite set. We have the equivalence:

$$
\operatorname{Supp}(f) \subset K \Longleftrightarrow R f(g)=0, \forall g \in \mathbb{G}: g \cap K=\varnothing \Longleftrightarrow \operatorname{Supp}(R f) \subset \bigcup_{x \in K} G_{x}
$$


This result is analogous to the support theorem in the Euclidean case [3].

\section{Acknowledgments}

I would like to thank Professor Ahmed Abouelaz for his guidance in this matter.

\section{References}

[1] I.M. Gelfand, M.I. Graev, and S.J. Shapiro, Differential forms and integral geometry, Funct. Anal. Appl. 3 (1969) pp. 24-40.

[2] F. Gonzalez, Radon transform on Grassmann manifolds, J. Funct. Anal. 71 (1987) pp. 339-362.

[3] S. Helgason, Integral Geometry and Radon Transforms, Springer, 2011.

[4] B. Rubin, Radon transforms on affine Grassmannians, Trans. Amer. Math. Soc. 356 (2004) pp. 5045-5070. 